\title{
Airlines Automatic Generation used in hostile environments
}

\author{
Ming $\mathrm{CHENG}^{1 \text {, a }}$, Yu Han $\mathrm{CHEN}^{2, \mathrm{~b}}$ \\ ${ }^{1}$ Naval War Gaming Center, Navy Command College, Nanjing, China \\ ${ }^{2}$ The 28th Research Institute, China Electronics Technology Group Corporation, Nanjing, China

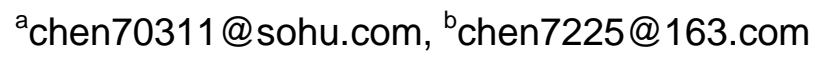

Keywords: Airline planning, ant colony algorithm, three-dimensional terrain, radar detection, fire probability.

Abstract. In a hostile environment, it is important for avoiding the threat of ground radar and ground air defense missile. So, in the airlines planning process these are some factors to be studied carefully, such as the terrain, ground radar and anti-aircraft missiles and other factors. Firstly, it is important to construct the battle environment spatial modeling. The battle space is divided by grid-like space nodes. Then set the values of modes attributes in the space node, such as the terrain model, radar model and anti-aircraft missiles model. The probability of detection was calculated based on the positional relationship between the radar and radar aircraft, and anti-aircraft missile shot probability, etc. Then the fitness function of a space node was gotten. Finally, using the ant colony algorithm to search the optimal airline, generate best performance of the airline.

\section{Introduction}

Automatic airline generation, also known as airline planning, it refers to use of computer information processing capabilities, get a optimal possible flight path based on consideration of aircraft maneuverability, penetration probability, topography and flight time constraints from the starting point to a target point[1]. In recent years, the radar detection and missile attacking technology has become more advanced, increasingly sophisticated air defense system in modern warfare. The automatic generation is main way to improve aircraft operational effectiveness, implement an effective means of long-range precision strike [2].

About the technology of airline planning, scholars had a lot of research, such as literature [4] use the A* algorithm for three-dimensional route planning. This method can enhance planning speed, but in a complex environment it is more difficult to obtain the optimal route. So it cannot be effectively applied. The literature[5] adopted PSO route search in a two-dimensional environment, to convert a variety of threats into the ground radar detection model, and circumvent the treats, which can better handle the situation in real-time multi-threats airlines planning. The literature [6] proposed genetic algorithms to route search, it can achieve adaptive route planning in an uncertain battlefield environment, but when in a large-scale environments, it is easy to fall into local optimum, not to get the best route. The complex battlefield situation needs to be adjusted quickly. The literature [7] although has strong flexibility algorithm, but there are slow search speed, not so easy to fall into local optimum global optimum route problem. For solving the problems of basic ant colony algorithm, the paper improved it by optimizes the fitness function and the using of heuristic search direction.

\section{Airline generation method}

With the improvement of the performance in air defense systems, such as radar detection capability, air defense missile firing accuracy and anti-jamming capability are all rapidly increased. The aircrafts are facing increasingly serious threats. Combat aircraft must be carefully avoid ground threats for survival, in order to ensure the safety of arriving the attack points. The airline generation is using computer information processing capabilities, comprehensive consideration of the flight time, fuel consumption, radar, air defense missile threat under space flight safety and other factors. Airlines can be generated automatically premise guarantee the safety of aircraft penetration of flight. 


\section{Airline automatic generation model}

Airline planning has constructed models include space model, radar model, electronic interference model, anti-aircraft missiles and fuel consumption models shoot model. The spatial modeling refers to the scope of the search space during airlines planning. Before airlines planning, flight environment (terrain, ground threats, etc.) must be expressed as identification information in order to be understood by the software.

For airline, the terrain is very important, the three-dimensional spatial airline planning dimensional mesh, each node in a spatial grid as an information space, each node can be expressed as $n_{\text {node }}=\left\{x, y, z, f_{\text {flag }}, f_{\text {dect }}, f_{\text {fire }}\right\}$, among them, xyz are three-dimensional grid coordinates for the location of information. $f_{\text {flag }}$ is grid properties flag. It expresses whether the plane can fly into this grid and or not, with 1 's and 0 's, such as the grid in the mountains inside, then represented by 0 , completely cannot fly; $f_{\text {dect }}$ is the probability of detection of radar and other sensors; $f_{\text {fire }}$ is the probability of being hit by anti-aircraft missiles. When the environment information changed, such as anti-aircraft missile sites were destroyed, the corresponding parameter to be up to date in order to be able to apply the latest real-time route planning and environmental data.

The size of the three-dimensional grid, also known as planning granularity space model, such as the grid $1 \times 1 \times 1(\mathrm{~km})$ is smaller than $2 \times 2 \times 2(\mathrm{~km})$ spatial grid. Generally speaking, the more dense grid, the more accurate route planning output, but the greater the amount, there are more calculation; the other hand, should take into account the maneuverability of the aircraft, so that the current grid and any adjacent space lattice spatial relations conforms to the airplane maneuvering capabilities, spatial grid setting cannot be too dense.

Airline planning model also contains the cost function for flight, the track can simply consider only the cost of ground radar detection probability and ground air defense missile fire probability, and the airline cost function calculation is divided into the following three steps:

Calculate the possibility of radar detection

The radar is the main system to detect, identify and track air goals in long-range. It is the main air defense warning system. Radar equation describes the basic characteristics of the radar system of mathematical equations [8].

$$
\mathrm{R}_{\max }=\left[\frac{\mathrm{P}_{\mathrm{T}} \mathrm{G}^{2} \lambda^{2} \sigma}{(4 \pi)^{\mathrm{s}} \mathrm{kT}_{0} \mathrm{~B}_{\mathrm{n}} \mathrm{F}_{\mathrm{n}}\left[\frac{\mathrm{S}}{\mathrm{N}}\right]_{\min } \mathrm{L}}\right]^{\frac{1}{4}}
$$

Where, $P_{\mathrm{T}}$ is radar transmitter pulse power; $\mathrm{G}$ is the radar antenna gain; $\lambda$ is the radar wavelength; $\sigma$ is RCS; $k$ is Boltzmann's constant; $T_{0}$ is working noise temperature; $B_{n}$ is Noise bandwidth; $F_{n}$ is Noise Figure; $(\mathrm{S} / \mathrm{N})_{\min }$ is the value of detecting input terminal minimum SNR required for detection; $\mathrm{L}$ is the total loss coefficient.

If taking into account the effects of electromagnetic interference, radar detection distances calculation [9].

$$
R_{t}=\left[\frac{P_{t} G_{t}^{2} \sigma K_{r}}{4 \pi B_{r}^{-S} L_{r}} \sum_{j=1}^{n} \frac{R_{j}^{2} B_{j} L_{j}}{P_{j} G_{j}(\theta) G_{r}(\varphi) \gamma_{j}}\right]^{\frac{1}{4}}
$$

Where, $K_{r}$ is radar jamming factor; $(s / J)$ is SIR; $R_{j}$ is the distance between the jammer and radar; $\mathrm{L}_{\mathrm{j}}$ is the jammer system losses; $\gamma_{\mathrm{j}}$ is the interference signal radar antenna polarization loss; $\mathrm{G}_{\mathrm{j}}(\theta)$ is gain on the radar jammer direction; $\mathrm{G}_{\mathrm{r}}(\varphi)$ is radar antenna gain in the direction of the jammer. $\Theta$ is the radar jammer angle error angle which is defined as a point jammer interference and jamming and radar connection; $\varphi$ is the radar main lobe pointing angles and radar and jammer connection. Relationship in probability of detection, the distance between the radar and the target is as follows.

$$
P=\frac{R^{4}}{r^{4}+R^{4}}
$$


Where, $\mathrm{P}$ is probability of radar detection; $\mathrm{R}$ is farthest distance radar detection; $\mathrm{r}$ is the distance between the target and the radar.

Calculate the possibility of being shot

The probability which the aircraft is being shot down by missiles is containing two parts [10-12]; the target missile interception event is the event A, anti-aircraft guns to intercept the target event is the event $\mathrm{B}$, combined with anti-aircraft missile and artillery firing system can be expressed. The probability is at least one anti-aircraft guns and missiles weapons to shoot down targets. Taking into account the timing and target shooting distribution, combined with air defense missile and artillery systems firing probability is $\mathrm{S}(\mathrm{AUB})=\mathrm{S}(\mathrm{A})+\mathrm{S}(\mathrm{B})-\mathrm{S}(\mathrm{AB})$;

$$
\begin{aligned}
& S(A)=P_{\left(t>t_{f m}+t_{r m}\right)}=\int_{t_{f m}+t_{r m}}^{\infty} \lambda_{1} e^{-\lambda_{1} t} d t \\
& \mathrm{~S}(\mathrm{~B})=\mathrm{P}_{\left(\mathrm{t}>\mathrm{t}_{\mathrm{fg}}+\mathrm{t}_{\mathrm{rg}}\right)}=\int_{\mathrm{t}_{\mathrm{fg}}+\mathrm{t}_{\mathrm{rg}}}^{\infty} \lambda_{2} \mathrm{e}^{-\lambda_{\mathrm{z}} \mathrm{t}} \mathrm{dt}
\end{aligned}
$$

Where, S(A) is the probability of Antiaircraft Missile Firing; S(B) is the antiaircraft fire probability; $\mathrm{S}(\mathrm{AB})$ is the anti-aircraft missiles and anti-aircraft guns shooting at the same time the probability of; $t_{f m}$ Air defense missile to intercept point desired event; $t_{r m}$ is the reaction time of anti-aircraft missiles; $\mathrm{t}_{\mathrm{fg}}$ is the projectile intercept flight to the desired point in time; $\mathrm{t}_{\mathrm{rg}}$ is the antiaircraft gun system response time; $\lambda_{1}$ is when anti-aircraft missiles to intercept corresponding to the average density of air strikes; $\lambda_{2}$ is the antiaircraft interception system corresponding to the average density of air strikes.

\section{The cost function}

For Airlines, the main consideration is to avoid the threat, so calculate the cost function is mainly considered threat radar and anti-aircraft missile threat, Route cost function $\mathrm{F}_{\text {line }}$ is the largest airline in radar detection probability and probability plot to shoot down missiles:

$$
F_{\text {line }}=\operatorname{Max}\left(\mathrm{PM}_{\mathrm{x}, \mathrm{y}, \mathrm{z}} \times \mathrm{PR}_{\mathrm{x}, \mathrm{y}, \mathrm{z}}\right)
$$

Where, $\mathrm{PM}_{\mathrm{x}, \mathrm{y}_{*}} \mathrm{z}$ is the probability shoot down missiles, $\mathrm{PR}_{\mathrm{x}, \mathrm{y}, \mathrm{z}}$ is the radar detection probability.

\section{Airlines planning based on ant colony algorithm}

Ant colony algorithm

During the search, the ants may use the cost of a small selection of local spatial grid to make the best track deviation. It may waste a lot of search event. Neural network training has been inspired by supervised learning methods, the introduction of the ideal output feedback, thus speeding up the learning speed of neural network, and make better track output close to the ideal. This paper introduces the deviation factor $\theta$, The off-angle feedback signals to the system as a search, speed up the search, but also easy to find the optimal solution, so heuristic information is used as follow:

$$
\eta=k / \theta
$$

Where, $\mathrm{k}$ is parameter.

In order to improve search efficiency of ants, establish a set of prior grids for searching in each spatial grid, the ants select next grid in this set. Establish of prior set is identify all spatial grid can fly around this grid are all set to prior search sets, and then to increase dynamically during operation ant colony algorithm based on the information on the concentration of each path reduce the number of search sets. This dynamic set of methods to establish the optimal search algorithm itself can benefit from ACO: pheromone concentration is not strong ant path is not easy to select. Also according to the distance and a spatial grid surrounding nodes are sorted using a fixed number of space grid method vicinity.

\section{Algorithm implementation}

Step 1: Spatial grid initialization information, optimal search space lattice initialize collection; Step 2: Pheromone spatial grid initialization information on an initial matrix; 
Step 3: The $M$ ants set in space grid starting point;

Step 4: According to $\eta$ and prior search set, move ant to spatial grid until all the ants reach the target point;

Step 5: Each ant computing airline cost function, recording the best performance of the current airline;

Step 6: Using the pheromone updating spatial grid along the airline biological pheromone strength;

Step 7: Repeat steps 2-5 until more than a predetermined number of iterations.

\section{The example analysis}

Suppose route planning space is the $100 \mathrm{~km}$ long, $100 \mathrm{~km}$ wide, $20 \mathrm{~km}$ height range, the range is divided into three-dimensional spatial lattice space of $100 \times 100 \times 20$, the mission area has a total of 200000 grid space. According to the three-dimensional spatial grid terrain data, set the value of $f_{\text {flag }}$, calculated the values of $f_{\text {fire }}$ and $f_{\text {dect }}$ for each grid based on the deployment of missile and radar positions deployment. Airlines automatically generated according to the above algorithm.

\section{Conclusions}

In order to quickly generate airline which can evade radar, ground missiles Air, some models were constructed, such as three-dimensional modeling of the battlefield environment. Then mark the radar and the air defense missile power, using the automatic generation method based on ant colony algorithm to generate comprehensive performance optimum airline. Finally after validate and analysis this method by example, it is proved that this method is reliable and effective.

\section{References}

[1] Tang Q, Zhang XL,Zuo 1. Initial Study on the Path Planning Algorithms for Unmanned Aerial Vehicles [J]. AERONAUTICAL COMPUTER TECHNIQUE. 2003. (01): 125-128.

[2] Min CW, Yuan JP. Introduction of Military Aircraft Route Planning[J]. FLIGHT DYNAMICS. 1998. (04): 16-21.

[4] Xiang-bin SHI, Yong-hao Y U, Dong-qi LIU,Shu-xia L I. The Algorithm of 3D-Route-Planning Based on A* [J]. Journal of Liaoning University(Natural Science Edition).2012，39(4): 295-299.

[5] Shang-qin TANG, Chang-qiang HUANG, Jie HU, Wen-chao WU. Threat equivalent and improved PSO algorithm based real-time method of UCAV route planning [J]. SYSTEMS ENGINEERING AND ELECTRONICS , 2010， 32(8): 1706-1710.

[6] Eun Y, Band H. Cooperative Task Assignment/Path Planning of Multiple Unmanned Aerial Vehicles Using Genetic Algorithms[J]. Journal of Aircraft, 2009, 46(1): 38-43.

[7] Zhen-feng LIU, Hong-sen XIE, Shui-gen WEI. Aircraft 3-D Route Planning Based on Culture-Ant Algorithm [J]. Computer Simulation, 2013, 30(5): 99-103.

[8] QIU Zhi-ping, LU Qiang-hua. Interval Estmation for Radar Detection Range[J]. AIRCRAFT DESIGN. 2008. (02): 1-5.

[9] CHEN Gan-tao, XU Jia, GAO Xiao, LIU Ming-lu, QU Jun-liang, NIE Ming-xin. Analysis and Calculation of Radar Detection Range with Active Blanket Jamming [J]. RADAR SCIENCE AND TECHNOLOGY. 2011. (01): 13-17.

[10] Ning WEI,Shi-ming ZHOU,Guo-hui DONG,Xue-min SONG. Shoot Probability in Aerial Defense Battle [J]. ORDNANCE INDUSTRY AUTOMATION. 2006. (12): 21-23. 
[11] Han Xing, Duan Fangfang, Gao Jian. Research on Firing Probability of Air Defense System [J]. Fire Control Radar Technology. 2014. (03): 26-28.

[12]CHEN Jian-hu, LIN Chong-yang. Tactical Mode of Shooting Probability Index for Ground-to-Air Missile Weapon System [J]. Armament Automation. 2007. (04): 12-13. 